\title{
APTITUD COMBINATORIA GENERAL Y ESPECÍFICA DE LÍNEAS DE MAÍZ (Zea mays) TROPICAL Y SUBTROPICAL ${ }^{1}$
}

\author{
Narciso Vergara Avila², Sergio A. Rodríguez Herrera ${ }^{3}$, Hugo S. Córdova Orellana ${ }^{2}$
}

\begin{abstract}
RESUMEN
Aptitud combinatoria general y específica de líneas de maíz (Zea mays) tropical y subtropical. Veinte líneas fueron cruzadas con seis líneas usadas como probadores, con el objetivo de examinar su aptitud combinatoria, general y específica, y determinar su utilización en un programa de hibridación. Las cruzas fueron evaluadas en tres localidades en México durante 1997. Estimaciones de aptitud combinatoria general (ACG) y aptitud combinatoria específica (ACE) fueron obtenidas usando un análisis de Línea x Probador. CML264 (1.586 kg/ha) y CML319 (1.285 kg/ha) tuvieron el mayor efecto de ACG en el grupo de líneas tropical y subtropical, respectivamente. El mayor efecto de ACE fueron para las cruzas CML322 x M.L.S4-1 (1.651 kg/ha) y CML258 x SSE255-18-19 (1.512 kg/ha). Estos resultados señalan que es posible estructurar un programa de híbridos utilizando las mejores líneas del Centro Internacional de Mejoramiento de Maíz y Trigo (CIMMYT) y los mejores probadores de la Universidad Autónoma Agraria Antonio Narro (UAAAN).
\end{abstract}

Palabras clave: Líneas, probadores, ACG, ACE,

\begin{abstract}
General and specific combining ability of tropical and subtropical maize (Zea mays) lines. Twenty maize lines were crossed with six inbreds used as testers, with the objective of examining their general and specific combining ability and determine their usefulness in a hybridization program. The crosses were evaluated in three environments at Mexico during 1997. General combining ability (GCA) and specific combining ability (SCA) effects were calculated using a Line $x$ Tester analysis. CML264 (1586 kg/ha) and CML319 (1285 kg/ha) were the highest GCA lines in the tropical and subtropical group, respectively. The highest SCA effects were for the crosses CML322 x M.L.S4-1 (1651 $\mathrm{kg} / \mathrm{ha})$ and CML258 x SSE255-18-19 (1512 kg/ha). These results indicate that it is possible to develop a hybrid program using the best lines released by the International Maize and Wheat Improvement Center (CIMMYT), and the best testers from UAAAN.
\end{abstract}

Key words: Lines, testers, GCA, SCA, hybrids. híbridos.

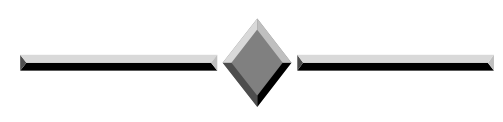

\section{INTRODUCCIÓN}

Las cruzas dialélicas generalmente son utilizadas por los programas de mejoramiento de plantas para obtener información de aptitud combinatoria y parámetros genéticos de sus fuentes de germoplasma. Sin embargo, éstas han sido usadas y abusadas más extensivamente que otros, causando una controversia acerca de su utilidad (Hallauer y Miranda 1981).

Griffing (1956) estableció cuatro métodos de diseños dialélicos para estimar la aptitud combinatoria general y específica de líneas, los cuales son adecuados cuando el número de progenitores es reducido, pero cuando este número se incrementa el procedimiento es más difícil. Para solucionar lo anterior, se propuso el método de mestizos, el cual utiliza una amplia base de genotipos usando un probador para determinar la habilidad combinatoria general de las líneas. Mientras que, el análisis de línea x probador, es una extensión del método anterior, en el cual varios probadores son utilizados, proporcionando información de aptitud combinatoria general y específica, y además estima varios tipos de efectos genéticos (Singh y Chaudhary 1985).

1 Recibido: 10 de noviembre, 2004. Aceptado: 30 de agosto, 2005. Presentado en la XLVI Reunión Anual del PCCMCA. San Juan, Puerto Rico. 2000.

2 Centro Internacional de Mejoramiento de Maíz y Trigo (CIMMYT), Apdo. Postal 6-641, 06600 México D.F., México. Email:nvergara58@hotmail.com, hcordova@cgiar.org.

3 Universidad Autónoma Agraria Antonio Narro, Instituto Mexicano del Maíz, Buenavista 25315, Saltillo, Coah. México.: E-mail: serroh99@hotmail.com 
La identificación de pares de líneas con comportamiento superior en rendimiento en combinaciones híbridas es una situación común en un programa de hibridación, en donde se deberá tener una serie de líneas disponibles las cuales se puedan cruzar con uno de los padres de una cruza simple élite con el propósito de aislar nuevas líneas a partir de generaciones segregantes, las cuales van a mejorar el comportamiento del híbrido original (Dudley 1984 y Dudley 1987).

En el programa de mejoramiento genético de maíz de la Universidad Autónoma Agraria Antonio Narro se ha estado usando germoplasma introducido como una fuente principal de heterosis en cruzas con el germoplasma local para obtener híbridos altamente productivos. El objetivo de este estudio es estimar los efectos de aptitud combinatoria general y específica de las líneas de CIMMYT, con el propósito de identificar las más sobresalientes y utilizarlas dentro del programa de mejoramiento genético de la UAAAN.

\section{MATERIALES Y MÉTODOS}

\section{Material genético}

Veinte líneas élites con un nivel de endogamia de $\mathrm{S}_{6}$ - $\mathrm{S}_{10}$ blancas, desarrolladas por el Centro Internacional de Mejoramiento de Maíz y Trigo (CIMMYT), y seis líneas élites del Instituto Mexicano del Maíz (IMM) de la Universidad Autónoma Agraria Antonio Narro (UAAAN), fueron seleccionadas para realizar este estudio (Cuadro 1). Las 20 líneas fueron cruzadas en forma manual y apareada con las seis líneas usadas como probadores durante la época de siembra en Tepalcingo, Morelos, 1997. Se establecieron dos fechas de siembra para procurar efectuar todas las cruzas posibles; sin embargo, no se pudo obtener semilla suficiente de 21 de las 120 posibles cruzas. En la cosecha se juntaron las mazorcas provenientes de cada cruza, desgranándose por separado para posteriormente hacer una mezcla de semillas de ambas cruzas con la finalidad de obtener suficiente semilla para representar cada cruza en particular.

\section{Evaluación de experimentos}

Ciento diecinueve cruzas (116 cruzas línea x probador, y tres cruzas entre probadores) y dos testigos locales, fueron evaluadas bajo un diseño experimental látice simple $11 \times 11$ con dos repeticiones, durante el verano de 1997, en Celaya, Guanajuato (1.754 msnm, $20^{\circ} 31^{\prime}$ ' latitud norte, $100^{\circ} 49^{\prime}$ longitud oeste); Tlalatizapan, Morelos (840 msnm, $18^{\circ} 41^{\prime}$ latitud norte, $99^{\circ}$ $68^{\prime}$ longitud oeste), y Cotaxtla, Veracruz (15 msnm, $18^{\circ}$ 50' latitud norte, $96^{\circ} 10^{\prime}$ longitud oeste) (García 1988).

Cuadro 1. Descripción del material utilizado en este estudio. México, 1997.

\begin{tabular}{llllll}
\hline Código & \multicolumn{1}{c}{ Germoplasma } & Adaptación & & Germoplasma & Adaptación \\
\hline & Líneas del (CIMMYT) & & & & \\
CML247 & Pool 24 & Tropical & CML311 & Población 500 & Subtropical \\
CML254 & Tuxpeño Sequía & Tropical & CML312 & Población 500 & Subtropical \\
CML258 & Población 21 & Tropical & CML313 & Población 501 & Subtropical \\
CML264 & Población 21 & Tropical & CML314 & Población 600 & Subtropical \\
CML271 & Población 29 & Tropical & CML315 & Población 500 & Subtropical \\
CML273 & Población 43 & Tropical & CML318 & Población 87 & Subtropical \\
CML275 & Población 43 & Tropical & CML319 & Población 89 & Subtropical \\
CML277 & Población 43 & Tropical & CML320 & Población 501 & Subtropical \\
CML278 & Población 43 & Tropical & CML322 & Población 89 & Subtropical \\
CML281 & Población 43 & Tropical & CML246 & Población 800 & V. Altos \\
& & & & & \\
AN1 & Probadores del (IMM) & & & & \\
AN2 & AN1 & Bajío & & & \\
SSE232 & SSE232-33-30 & Bajío & & & \\
SSE255 & SSE255-18-19 & Bajío & & & \\
SSE255 & SSE255-18-19 & Bajío & & & \\
M.L.S4-1 & M.L.S4-1 & Bajío & & & \\
\hline
\end{tabular}

CML = Líneas liberadas por CIMMYT. 
La parcela experimental consistió de un surco de cinco metros de largo espaciados a $75 \mathrm{~cm}$, con una distancia de siembra entre plantas de $25 \mathrm{~cm}$, depositándose dos semillas por punto de siembra para posteriormente ralear a una planta y así obtener una densidad de 53.333 plantas por hectárea. Se registraron datos de rendimiento de grano el cual fue ajustado al $15,5 \%$ de humedad y $85 \%$ de desgrane, así como otras características agronómicas importantes, como días a floración masculina y femenina, altura de planta y mazorca, acame de raíz y tallo, pudrición y cobertura de mazorca.

\section{Análisis estadísticos}

El comportamiento para rendimiento de las cruzas faltantes se predijo utilizando el método propuesto por Eckhardt (1951). Los valores estimados de rendimiento se promediaron junto con los rendimientos de las otras cruzas para obtener un estimador de ACG (aptitud combinatoria general) que fuera más confiable y preciso. Después de la predicción de las cruzas faltantes, en cada localidad se realizó un análisis de varianza con base en el modelo del diseño látice propuesto por Gómez y Gómez (1984), usando el paquete estadístico Alfa - Látice (Barreto et al. 1993). Los programas PROC GLM (SAS 1997) y PROC MIXED (Littell et al. 1996), fueron usados para calcular los componentes de varianza y las pruebas de $\boldsymbol{F}$ en el análisis de varianza combinado, donde ambientes y repeticiones fueron consideradas como efectos aleatorios y las cruzas como fijos. Los programas PROC MIXED y LSMEANS de SAS fueron usados para obtener las medias ajustadas de rendimiento de grano a través de repeticiones y ambientes.

Se realizó un análisis genético línea x probador para cada localidad y a través de localidades para rendimiento de grano usando el método descrito por Kempthorne (1957), lo cual permitió estimar los efectos de ACG y ACE para los progenitores y sus cruzas. Dichos efectos, fueron probados usando una prueba de $\boldsymbol{t}$, utilizando sus valores de error estándar (Singh y Chaudhary 1985). Las pruebas de $\boldsymbol{F}$ para el análisis combinado (Línea x Probador), fueron calculadas de la siguiente manera: los efectos principales líneas y probadores fueron probadas contra sus respectivas interacciones con localidades, las demás interacciones fueron probados contra la triple interacción y ésta a su vez fue probada contra el error experimental. Además, se construyó la estructura genética para rendimiento de grano de cada una de las 120 cruzas con base a los efectos de ACG y ACE, de acuerdo a la metodología descrita por Claure (1990), con el fin de observar si el alto rendimiento de ciertas cruzas se debe a: efectos aditivos $(g i+g j)>S i j$; efectos no aditivos $S i j>$ $(g i+g j)$ o a efectos aditivos más no aditivos $(g i+g j)=S i j$.
Sobre la base del modelo $(Y i j=u+g i+g j+S i j)$

Donde:

$Y i j=$ rendimiento promedio de una cruza en el que interviene el ij-ésimo progenitor.

$u=$ valor medio del grupo de cruzas neas)

$g i=$ efecto de ACG del i-ésimo progenitor (Lídores

$g j=$ efecto de ACG del $\mathrm{j}$-ésimo progenitor (Proba-

Sij $=$ efecto de ACE del i-ésimo progenitor con el $\mathrm{j}$-ésimo probador.

Sobre la base de los efectos de ACG de las líneas, se procedió a la clasificación en valores altos, intermedio y bajo, mediante la agrupación de los valores de ACG $>1$, ACG cercanos a 1, y ACG $<1$, respectivamente.

\section{RESULTADOS Y DISCUSIÓN}

Se encontraron diferencias altamente significativas en todas las fuentes de variación en el análisis de varianza combinado para rendimiento de grano (Cuadro 2). Las diferencias observadas en localidades pueden ser atribuidas a las condiciones climáticas; Cotaxtla es una localidad de clima tropical de la costa del Golfo de México y se encuentra a nivel del mar; Tlaltizapan tiene clima subtropical con una altitud de $840 \mathrm{msnm}$, y Celaya está más distante de éstas a una altitud de 1.754 msnm y es considera como Bajío. Las diferencias

Cuadro 2. Análisis de varianza combinado (línea x probador) para rendimiento de grano (kg/ha). México, 1997.

\begin{tabular}{lrrr}
\hline $\begin{array}{c}\text { Fuentes de } \\
\text { variación }\end{array}$ & $\begin{array}{c}\text { Grados de } \\
\text { libertad }\end{array}$ & $\begin{array}{c}\text { Cuadrados } \\
\text { medios }\end{array}$ & \multicolumn{1}{c}{$\begin{array}{c}\text { F } \\
\text { calculada }\end{array}$} \\
\hline Localidad (Loc.) & 2 & 2659602114,9 & $1.936,0 * *$ \\
LC (ACG) & 19 & 30856126,4 & $6,1 * *$ \\
LN (ACG) & 5 & 2832560,7 & $5,6 * *$ \\
LC x LN (ACE) & 95 & 5074887,3 & $2,2 * *$ \\
LC (ACG) x Loc. & 38 & 7966543,4 & $3,4 * *$ \\
LN (ACG) x Loc. & 10 & 21936303,2 & $9,3 * *$ \\
LC x LN (ACE) x Loc. & 190 & 2361707,1 & $1,7 * *$ \\
Error & 360 & 1373748,7 & \\
Total & 719 & & \\
DMS (0,05) & & $1.326,3$ & \\
C.V (\%) & \multicolumn{3}{c}{12,5} \\
\hline
\end{tabular}

*, ** Diferencias significativas a 0,05 y $0,01 \%$ de probabilidad. LC = líneas de CIMMYT; LN = líneas de NARRO. 
observadas en las líneas (LC) y probadores (LN), se deben a que provienen de diferente germoplasma y tienen diferente adaptación climática. En la interacción LC x $\mathrm{LN}$, las diferencias encontradas indican que las líneas de CIMMYT clasifican a la ACE de las líneas de NARRO en forma diferente, y viceversa. Estos resultados eran de esperarse debido a que existen diferencias dentro de cada grupo coincidiendo con lo reportado por Hallauer (1990). Las diferencias en la interacción de líneas (LC) y probadores (LN) con localidades, indican que ambos grupos tuvieron un comportamiento diferente en cada una de las localidades de evaluación. Las diferencias en la triple interacción LC x LN x localidad, muestran que los materiales se comportaron de manera diferente de una localidad a otra.

Los estimadores de los efectos de ACG para ambos grupos de líneas en cada localidad y a través de localidades se muestran en el Cuadro 3. En el grupo de

Cuadro 3. Efectos de aptitud combinatoria general (ACG) por localidad y a través de localidades para rendimiento de grano $(\mathrm{kg} / \mathrm{ha})$ de los dos grupos de líneas evaluadas. México, 1997.

\begin{tabular}{|c|c|c|c|c|}
\hline Línea & Celaya & Tlaltizapan & Cotaxtla & Combinado \\
\hline \multicolumn{5}{|l|}{ CIMMYT } \\
\hline CML247 & $-2166,6 *$ & $-1134,8 *$ & $-643,3 *$ & $-1314,9 *$ \\
\hline CML254 & $-690,9$ & $-45,1$ & $613,6^{*}$ & $-40,8$ \\
\hline CML258 & 616,4 & $1036,4 *$ & $1657,5 *$ & $1103,4 *$ \\
\hline CML264 & $2685,8 *$ & $1154,2 *$ & $918,5 *$ & $1586,2 *$ \\
\hline CML271 & $-1212,6 *$ & $-748,8 *$ & 291,4 & $-556,7$ \\
\hline CML273 & $1822,1 *$ & 130,3 & 268,0 & 740,1 \\
\hline CML275 & $-1849,5 *$ & $-907,6 *$ & $-524,1$ & $-1093,8 *$ \\
\hline CML277 & 321,4 & 379,5 & $1292,5 *$ & 664,5 \\
\hline CML278 & $-632,0$ & 60,5 & $-279,2$ & $-283,6$ \\
\hline CML281 & $1189,9 *$ & $1112,2 *$ & $1197,3 *$ & $1166,5 *$ \\
\hline CML311 & 281,4 & $-568,9 *$ & $953,4 *$ & 222,0 \\
\hline CML312 & $1210,0 *$ & $1040,0 *$ & $-95,3$ & 718,2 \\
\hline CML313 & $-365,8$ & $-403,2$ & $1391,1 *$ & 207,3 \\
\hline CML314 & $-52,7$ & 213,3 & 139,2 & 99,9 \\
\hline CML315 & $-881,0 *$ & $-258,3$ & $-1198,4 *$ & $-779,2$ \\
\hline CML318 & 198,9 & 210,1 & $-2269,7 *$ & $-620,2$ \\
\hline CML319 & $1241,7 *$ & $1031,5 *$ & $1580,7 *$ & $1284,6 *$ \\
\hline CML320 & $-538,7$ & $-113,3$ & $-1702,5 *$ & $-784,8$ \\
\hline CML322 & $-1152,00 *$ & $-814,6 *$ & $-311,7$ & $-759,4$ \\
\hline CML246 & $-25,9$ & $-1373,4 *$ & $-3278,9 *$ & $-1559,4^{*}$ \\
\hline Error estándar & 438,7 & 235,1 & 280,4 & 458,6 \\
\hline \multicolumn{5}{|l|}{ NARRO } \\
\hline AN1 & $-1877,8 *$ & $-346,8 *$ & $-50,2$ & $-758,3$ \\
\hline AN2 & $-227,3$ & $-968,7 *$ & $312,6 *$ & $-294,5$ \\
\hline SSE232-33-30 & $1116,9 *$ & $595,7 *$ & $-147,0$ & 521,9 \\
\hline SSE255-18-19 & $-468,9 *$ & 178,4 & 158,4 & $-44,1$ \\
\hline SSE255-18-19 & $-14,0$ & $277,4 *$ & 2,6 & 88,7 \\
\hline M.L.S4-1 & $1471,1 *$ & $264,0 *$ & $-276,5$ & 486,2 \\
\hline Error estándar & 225,1 & 120,6 & 143,9 & 390,3 \\
\hline
\end{tabular}

* Más grande que 2 x Error estándar. líneas de CIMMYT (LC), 10 líneas tuvieron efectos de ACG positivos, y las mejores líneas tropicales con alto valor de ACG positivo y significativo fueron CML264 (1.586,2 kg/ha), CML281 (1.166,5 kg/ha) y CML258 (1.103,4 kg/ha), mientras que CML319 (1.284,6 kg/ha) y CML312 (718,2 kg/ha) fueron las únicas líneas subtropicales con alto valor de ACG. Por su parte en el grupo de líneas de NARRO (LN) SSE232-33-30 (522 $\mathrm{kg} / \mathrm{ha})$ y M.L.S4-1 (486,2 kg/ha) fueron las mejores líneas con el mayor efecto de ACG positivo.

De acuerdo a la clasificación de los efectos de ACG (Figura 1), las líneas CML264, CML281, CML258 y CML319 fueron consideradas como de alta ACG; mientras que CML273, CML277 y CML312 fueron clasificadas de intermedia ACG y el resto de las líneas fueron consideradas como de baja ACG. Varios investigadores Betrán et al. (1997), Vergara et al. (1997), han confirmado el potencial genético de éstas líneas señalando su valor genético en combinaciones híbridas.

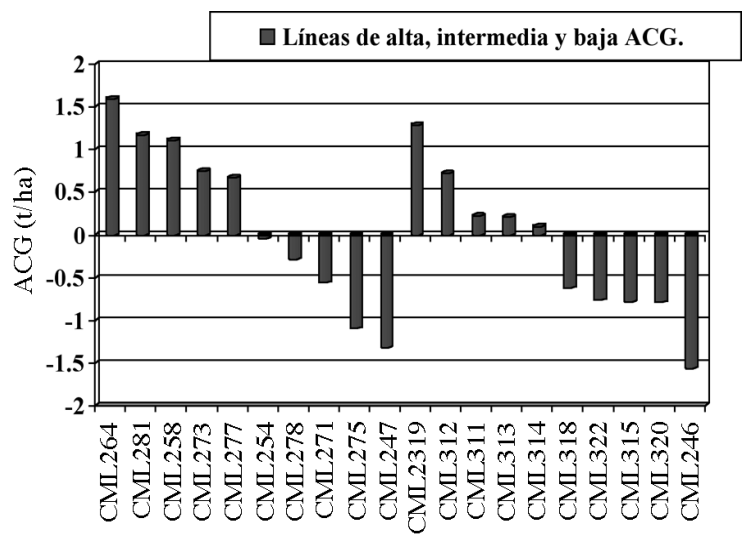

Figura 1. Clasificación de 10 líneas tropicales y 10 subtropicales de alta, intermedia y baja ACG. México, 1997.

Las cruzas CML258 x SSE255-18-19 (1512,21 $\mathrm{kg} / \mathrm{ha}$ ) y CML278 x AN2 (1.318,6 kg/ha), CML247 x SSE232-33-30 (1.257,7 kg/ha), CML247 x AN2 $(1.106,8 \mathrm{~kg} / \mathrm{ha})$ y CML275 x AN2 $(1.105,2 \mathrm{~kg} / \mathrm{ha})$, que involucran líneas tropicales tuvieron altos valores de ACE. Mientras que en las cruzas donde intervienen líneas subtropicales, CML322 x M.L.S4-1 (1.650,9 kg/ha), CML315 x AN2 (1.413 kg/ha), CML315 x M.L.S4-1 (1.301,1 kg/ha), CML320 x M.L.S4-1 (1.318,3 kg/ha) y CML318 x SSE255-18-19 (1.290,1 $\mathrm{kg} / \mathrm{ha}$ ) tuvieron alta ACE, destacándose CML247 y CML315 las cuales mostraron altos efectos de ACE con dos probadores, respectivamente (Cuadro 4). 
Cuadro 4. Efectos de aptitud combinatoria específica (ACE) a través de localidades para rendimiento de grano $(\mathrm{kg} / \mathrm{ha})$ de 20 líneas y seis probadores. México, 1997.

\begin{tabular}{lcccccc}
\hline & AN1 & AN2 & SSE232 & SSE255 & SSE255 & M.L.S4-1 \\
\hline CML247 & 255,5 & $1.106,8$ & $1.257,7^{*}$ & $-1000,5$ & $-1.133,2^{*}$ & $-486,2$ \\
CML254 & $-373,9$ & $1.074,8$ & $1.170,2^{*}$ & $-626,1$ & $-758,8$ & $-486,2$ \\
CML258 & $-675,3$ & $-440,5$ & $-1.289,7^{*}$ & $1512,2^{*}$ & $1.379,5^{*}$ & $-486,2$ \\
CML264 & 758,3 & $-320,9$ & 394,8 & 190,1 & 175,7 & $-1.197,9^{*}$ \\
CML271 & 617,9 & $-641,6$ & $-699,2$ & 417,7 & 285,0 & 20,1 \\
CML273 & $-305,1$ & $-897,8$ & 676,6 & 584,2 & 428,4 & $-486,2$ \\
CML275 & $-1.905,9^{*}$ & 1105,2 & 595,7 & 394,7 & 480,0 & $-669,8$ \\
CML277 & 845,4 & $-141,4$ & 357,3 & $-785,2$ & $-356,0$ & 79,8 \\
CML278 & 508,7 & $1318,6^{*}$ & 487,4 & $-1.046,4$ & $-1.179,2^{*}$ & $-89,1$ \\
CML281 & 758,2 & $-779,4$ & 255,0 & $-1.128,3^{*}$ & 14,5 & 880,1 \\
CML311 & 578,6 & $-305,4$ & $-399,0$ & 306,9 & 174,1 & $-355,2$ \\
CML312 & 416,6 & $-1.717,4^{*}$ & 70,0 & 126,6 & 799,1 & 305,0 \\
CML313 & $-578,6$ & 938,8 & 651,4 & $-38,5$ & $-54,4$ & $-918,8$ \\
CML314 & 794,1 & 115,2 & 747,5 & $-586,7$ & $-844,8$ & $-225,3$ \\
CML315 & $-2.267,3^{*}$ & $1.413,3^{*}$ & $-521,9$ & 103,7 & $-29,0$ & $1.301,1 *$ \\
CML318 & 758,3 & $-1.911,9 *$ & $-1.002,0$ & 509,8 & $1.290,1^{*}$ & 355,8 \\
CML319 & 758,3 & 311,2 & $-679,1$ & 402,3 & $-1.209,5 *$ & 416,9 \\
CML320 & $-1.104,1$ & 292,8 & $-637,3$ & $-321,2$ & 451,4 & $1.318,3^{*}$ \\
CML322 & $-900,8$ & $-1343,3^{*}$ & $-332,7$ & 525,0 & 401,0 & $1.650,9^{*}$ \\
CML246 & $1.061,1$ & 823,0 & $-1102,8$ & 459,8 & $-313,9$ & $-927,2$ \\
\hline
\end{tabular}

Error

estándar $\quad 558,2$

* Más grande que 2 x Error estándar.

El valor más alto de ACE fue registrado por la cruza CML322 x M.L.S4-1 (1.650,9 kg/ha); sin embargo, obtuvo el lugar diecisiete para rendimiento de grano $(10.758,3 \mathrm{~kg} / \mathrm{ha})$. Por su parte, la cruza CML258 x
SSE255-18-19 obtuvo el segundo lugar para ACE $(1.512,2 \mathrm{~kg} / \mathrm{ha}) \mathrm{y}$ primer lugar para rendimiento de grano (11.952,2 kg/ha) (Cuadro 5). Se puede observar que en las mejores cinco cruzas con valores altos de ACE,

Cuadro 5. Efectos de aptitud combinatoria específica (ACE) para rendimiento ( $\mathrm{kg} / \mathrm{ha})$, y su estructura genética de cinco cruzas superiores y cinco inferiores a través de localidades. México, 1997.

\begin{tabular}{lrrrrrrr}
\hline \multicolumn{1}{c}{ Cruza } & $\begin{array}{c}\text { Rend } \\
\text { (kg/ha) }\end{array}$ & Línea & Probador & gi+gj & ACE & $\dagger$ & + \\
\hline CML322 x M.LS4-1 & $10.758,3$ & $-759,4$ & 486,2 & $-273,2$ & $1.650,9$ & 1 & 17 \\
CML258 x SSE255-18-19 & $11.952,2$ & $1.103,4$ & $-44,1$ & $1.059,3$ & $1.512,2$ & 2 & 1 \\
CML315 x AN2 & $9.720,2$ & $-779,2$ & $-294,5$ & $-1.073,7$ & $1.413,3$ & 3 & 50 \\
CML278 x AN2 & $10.121,2$ & $-283,6$ & $-294,5$ & $-578,6$ & $1.318,6$ & 4 & 37 \\
CML320 x M.LS4-1 & $10.400,3$ & $-748,8$ & 486,2 & $-262,9$ & $1.318,3$ & 5 & 29 \\
CML322 x AN2 & $6.983,4$ & $-759,4$ & $-294,5$ & $-1.053,9$ & $-1.343,3$ & 116 & 115 \\
CML312 x AN2 & $8.087,0$ & 718,2 & $-294,5$ & 423,8 & $-1.717,4$ & 117 & 104 \\
CML275 x AN1 & $5.622,7$ & $-1.093,8$ & $-758,3$ & $-1.852,0$ & $-1.905,9$ & 118 & 119 \\
CML318 x AN2 & $6.554,0$ & $-620,2$ & $-294,5$ & $-914,7$ & $-1.911,9$ & 119 & 118 \\
CML315 x AN1 & $5.576,9$ & $-779,2$ & $-758,3$ & $-1.537,5$ & $-2.267,3$ & 120 & 120 \\
DMS (0,05) & $1.323,3$ & & & & & & \\
\hline
\end{tabular}

$\dagger=$ lugar ocupado según su valor de ACE.

$\$=$ lugar ocupado según su valor de rendimiento. 
Cuadro 6. Comportamiento promedio de cruzas con alto y bajo rendimiento $(\mathrm{kg} / \mathrm{ha})$, y su estructura genética a través de localidades. Verano, 1997 B. México, 1997.

\begin{tabular}{lrrrrrrr}
\hline \multicolumn{1}{c}{ Cruza } & $\begin{array}{c}\text { Rend. } \\
\text { (kg/ha) }\end{array}$ & Línea & Probador & gi+gj & ACE & $\dagger$ & + \\
\hline CML258 x SSE255-18-19 & $11.952,2$ & $1.103,4$ & $-44,2$ & $1.059,3$ & $1.512,2$ & 2 & 1 \\
CML281 x M.L.S4-1 & $11.913,3$ & $1.166,5$ & 486,2 & $1.652,7$ & 880,0 & 16 & 2 \\
CML264 x SSE232-33-30 & $11.883,5$ & $1.586,2$ & 521,9 & $2.108,1$ & 394,8 & 45 & 3 \\
CML319 x M.L.S4-1 & $11.568,4$ & $1.284,6$ & 486,2 & $1.770,8$ & 416,9 & 41 & 4 \\
CML281 x SSE232-33-30 & $11.323,9$ & $1.166,5$ & 521,9 & $1.688,4$ & 255,0 & 55 & 5 \\
CML322 x AN1 & $6.962,1$ & $-759,4$ & $-758,3$ & $-1.217,7$ & $-900,8$ & 102 & 116 \\
CML320 x AN1 & $6.733,5$ & $-784,8$ & $-758,3$ & $-1.543,1$ & $-1.104,1$ & 109 & 117 \\
CML318 x AN2 & $6.554,0$ & $-620,2$ & $-294,5$ & $-914,7$ & $-1.911,9$ & 119 & 118 \\
CML275 x AN1 & $5.622,7$ & $-1093,8$ & $-758,3$ & $-1.852,0$ & $-1.905,9$ & 118 & 119 \\
CML315 x AN1 & $5.576,9$ & $-779,2$ & $-758,3$ & $-1.537,5$ & $-2.267,3$ & 120 & 120 \\
DMS (0.05) & $1.323,3$ & & & & & & \\
\hline
\end{tabular}

$\dagger=$ lugar ocupado según su valor de ACE

$\$=$ lugar ocupado según su valor de rendimiento.

los efectos no aditivos fueron más importantes; con excepción de CML258 x SSE255-18-19, donde aparentemente ambos efectos aditivos y no aditivos fueron de igual importancia. Además en las cinco cruzas con los valores más bajos y negativos de ACE, ambos efectos aditivos y no aditivos fueron negativos.

Las cinco mejores cruzas tuvieron un rango de rendimiento de grano de 11.323,9 a 11.952,2 kg/ha, este alto rendimiento se debió a que los efectos aditivos fueron más importantes; excepto en la cruza CML258 x SSE255-18-19 donde los efectos no aditivos fueron más importantes (Cuadro 6). Por su parte, el bajo rendimiento de las cinco peores cruzas se debió a que ambos efectos aditivos y no aditivos fueron negativos.

\section{CONCLUSIONES}

Las líneas tropicales CML258, CML264, CML273, CML277, CML281 y subtropicales CML312 y CML319, mostraron los mejores efectos de ACG por lo que deberán ser consideradas como progenitores potenciales para formar híbridos con las líneas de la UAAAN. Las cruzas CML322 x M.L.S4-1 (1.651 kg/ha) y CML258 x SSE255-18-19 (1.512 kg/ha), tuvieron los mayores efectos de ACE.

Se observaron importantes combinaciones heteróticas entre las cruzas de las líneas de CIMMYT con las líneas del IMM usadas como probadores, las cuales pueden ser explotadas para el desarrollo de nuevos híbridos en un tiempo corto. Con los resultados de este estudio es posible diseñar y estructurar un programa de híbridos en el IMM, para incorporar el germoplasma de CIMMYT.

\section{LITERATURA CITADA}

BARRETO, H.; EDMEADES, G.; CHAPMAN, S.; CROSSA, J. 1993. El diseño Alfa-Látice en fitomejoramiento y agronomía: generación y análisis. Síntesis de resultados experimentales del Programa Regional de Maíz (PRM) 4: 273-283.

BETRÁN, F.; BECK, D.; EDMEADES, G.O.; RIBAUT, J.; BAZINGER, M.; SÁNCHEZ, C. 1997. Genetic analysis of abiotic stress tolerance in tropical maize hybrids. In: CIMMYT ed. Book of abstracts. The Genetic and Exploitation of Heterosis in Crops. An International Symposium. Mexico, D.F., Mexico. p. 28-29.

CLAURE I., V. 1990. Aumento del potencial de rendimiento mediante alternancia de híbridación y selección en maíz (Zea mays L.). Tesis doctoral, Colegio de postgraduados. Montecillos, México. 136 p.

DUDLEY, J. 1984. A method of identifying lines for use in improving parents of a single cross. Crop Sci. 24: 355357.

DUDLEY, J. 1987. Modification of methods for identifying inbred lines useful for improving parents of a elite single crosses. Crop Sci. 27:944-947. 
ECKHARDT, R. 1951. Predicting yields of missing single crosses of corn. Agron. J. 44: 215-216.

GARCÍA, E. 1988. Modificaciones al sistema de clasificación climática de Koppen (para adaptarlo a las condiciones de la república mexicana) 4 ed. UNAM. Instituto de Geografía. México, 217 p.

GÓMEZ A.; GÓMEZ, A.K. 1984. Statistical procedures for agricultural research. $2^{\text {nd }}$ ed. Wiley. USA. $680 \mathrm{p}$.

GRIFFING, B. 1956. Concept of general and specific combining ability in relation to diallel crossing systems. Aust. J. Biol. Sci. 9:463-493.

HALLAUER, A.; MIRANDA, J. 1981. Quantitative genetics in maize breeding. Iowa State University Press Ames, Iowa, USA. 468 p.

HALLAUER, A. 1990. Methods used in developing maize inbreds. Maydica 35: 1-16.
KEMPTHORNE, O. 1957. An introduction to genetics statistics. New York: Wiley, Inc. London. Chapman, Hall, Ltd. p. 468-471.

LITTELL, R.; MILLIKEN, G.; STROUP, W.; WOLFINGER, R. 1996. SAS system for mixed models. SAS Institute., cary, N.C. USA.

SAS. 1997. Statistical analysis system. SAS / STAT User's Guide: release 6.11. SAS Institute Inc. Cary, N. C. USA.

SINGH, R.; CHAUDHARY, B. 1985. Biometrical techniques in genetics and breeding. International Bioscience Publishers Hissar. India. p. 205-214.

VERGARA, A.; VASAL, S.; MCLEAN, S.; SRINIVASAN, G.; RODRÍGUEZ H. 1997. Heterosis and combining ability among long and short-ear maize inbred lines. In: CIMMYT ed. Book of abstracts. The Genetic and Exploitation of Heterosis in Crops. An International Symposium. Mexico, D.F., Mexico. p. 198-199. 